\title{
Caffeine and exercise join forces against skin cancer
}

Combining exercise with a low dose of caffeine can reduce the risk of skin cancer in mice, according to researchers at Rutgers University (New Brunswick, NJ). In previous mouse studies, Allan H. Conney and colleagues found that caffeine ingestion or voluntary exercise on a running wheel reduced mouse tissue fat and increased apoptosis in response to UVB exposure. Now, they show that a combination of running and caffeine has a greater effect than either treatment on its own (Proc. Natl. Acad. Sci.104, 12936-12941; 2007).

Over the course of two weeks, Conney's team gave mice caffeinated drinking water, voluntary access to a running wheel or a combination of the two. Mice treated with caffeine received either a low dose $(0.1 \mathrm{mg} /$ $\mathrm{ml})$ or a high dose $(0.4 \mathrm{mg} / \mathrm{ml})$. While none of the treatments significantly affected mice's overall body weight, all reduced the thickness of their tissue fat and increased apoptosis in sunburn cells following exposure to UVB lamps. The combination treatments had a greater effect than caffeine or voluntary exercise alone. This is not because mice on caffeine exercised more than their caffeinefree counterparts; all mice with running wheels ran approximately two miles per day. Notably, a low dose of caffeine combined with exercise had a greater-than-additive effect on UVB-induced apoptosis, and was more effective than the high-caffeine-dose/ exercise regimen. Conney and his group hope to shed light on this result in future doseresponse trials.

According to previous research by the Rutgers team, caffeine and exercise prematurely increase the level of cyclin B1 in the epidermis of UVB-treated mice, which may explain the pro-apoptotic effects of these regimens. Cyclin B1 has an important role in controlling cells' transition to mitosis. By raising the level of expression of this protein, exercise and caffeine may help override the $G_{2} / M$ checkpoint, causing cells to enter lethal mitosis and die. In the current study, combining caffeine and exercise also increased mice's epidermal level of $\mathrm{p} 53$ phosphorylated at serine-15, a protein associated

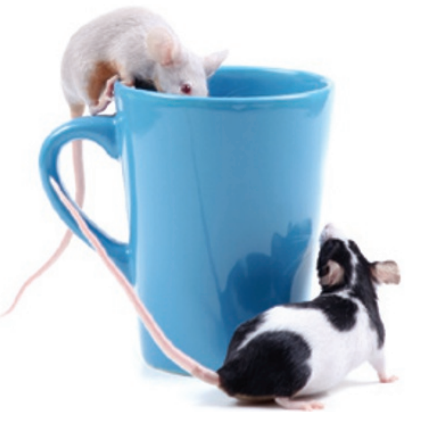

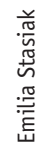

with tumor suppression. This did not occur when mice were treated with caffeine or exercise alone, which may account for the greater-than-additive effect of the combination treatment. Further studies are planned to elucidate the mechanism underlying these effects.

It remains to be seen whether humans would benefit from a cup of coffee and a jog before heading to the beach. "From what we already know, I think that the combination will be effective," Conney tells Lab Animal, "but someone needs to do the study."

Karen Marron

\section{HOW A MEMORY IS MADE}

Scientists claim to have visualized for the first time the cellular process of memory formation in rats. Gary Lynch and colleagues at the University of California (Irvine) and Carnegie Mellon University (Pittsburgh, PA) used microscopic technology and a special marker to observe memory-related changes in the synapses of rat brains.

A process called long-term potentiation (LTP) underlies these synapse changes. LTP is a transient increase in the strength of a synapse that is thought to contribute to memory formation and storage. Lynch's lab previously showed that induction of LTP in vitro was accompanied by a marked, temporary increase in the phosphorylation of cofilin, an actin-binding protein, in dendritic spines of the potentiated synapses. Now, the group has used cofilin phosphorylation as a biochemical marker of LTP induction in vivo.

Lynch and colleagues allowed one group of rats to explore a new environment and compared them with another group of rats that did not explore. After killing the rats, they measured cofilin phosphorylation in the hippocampus (J. Neurosci. 27, 8031$8039 ; 2007)$. The explorer rats had $30 \%$ more dendritic spines with phosphorylated cofilin than the other group. The incidence of spines containing phosphorylated cofilin was low overall
( 1 in 300). The hippocampal synapses with the LTP marker were $50 \%$ larger than those without.

The connection between memory and phosphorylated cofilin (and, by extension, LTP) was reinforced by results from rats that were given an NMDA receptor antagonist, which blocks the LTP process, before exploring. This treatment eliminated both memory formation and the increase in spines bearing phosphorylated cofilin.

The authors concluded that the links between phosphorylated cofilin and LTP, and between LTP and memory, suggest that the synapse changes observed in the study are directly related to the encoding of new information. They note that other mechanisms are probably responsible for long-term storage of that information.

Their ability to identify the few spines that change among the many that do not might be the first step toward mapping a complete memory circuit, a fundamental objective in learning and memory research. This goal will require even more powerful techniques, including the abilities to look at larger fields in more animals, and to look at later events. Lynch and his group now plan to apply this technique to identifying other areas of the brain that might be involved in memory.

Monica Harrington 